\title{
Disability and Rehabilitation Database in Chinese Language
}

\author{
Li Quankai and Guo Ming \\ China Rehabilitation Research Centre, Beijing, China
}

\begin{abstract}
Disability and Rehabilitation database collected articles published in China 's journals, newspapers, papers in conference and laws which were relevant to rehabilitation and related areas. These were in Chinese and published from the year of 1949 to 1993 . The DR database collected 35,000 records.
\end{abstract}

\section{Introduction}

Although the number of databases made in China probably exceeds 10,000 , the number of dababases relevant to any one field is substantially less. For a number of field, databases have been made. To the best of our knowledge, no such database has been made for the field of rehabilitation.

The records published in 400 journals , 100 newspapers, conference papers and laws on a specific field are collected in DR database.

The purpose of this communication is to provide a database which might help individuals 's searches for information relevant to rehabilitation in China.

\section{Method}

CDS/ISIS ( Computerized Docurment Service/ Integrated Set of Information System ) was used to make RD database. The operating enviroment: (1) Main memory of $1 \mathrm{MB}$, hard disk more than 40MB. (2)286 and above PC IBM and compatable.Struturing data design of the database consisted of 4 files : FDT( field definition table),FMT (format), FST (field select table), PFT (print format table). Once the four files were produced, the database was generated.

The first step to make the database was to select out 400 journals and 100 newspapers and then marked out every article which was relevant to rehabilitation. These journals and newspapers seem to indicate a wider range of coverage, including titles from such fields as orthopaedics,physical therapy, prosthetics and orthotics, physiology, bilological engineering, special education, base-community rehabilitation, disablility law and etc.

The second step was to fill worksheet. The worksheet consisted of 30 fields which had been definited in FDT. The most important fields were Chinese thesaurus, provisional headings. Chinese thesaurus were standard vocabulary terms that described the concepts covered in DR database records. Chinese thesaurus allowed 
users to retrieve all references to a particular topic, even if different terminology was used in the records. Each record contains several Chinese thesaunus. These thesaurus were found in the two CT fields : Major CT and Minor CT. Used the CT field abbreviation to search both CT fields. Used the MICT field abbreviation to find all records in which the term is a major topic. Used the MICT field abbreviation to find all records in which the term was a minor topic. All CT appeared in the CT fields. Major CT were marked with an asterisk. Since CT was not enough to cover the field of rehabilitation, provisional headings were added from Mesh (Medical Subject Headings) which were translated into Chinese and some special words we collected in our dailywork before. The third step was to input all these datas on worksheet into format in the database.

When these research was performed, a database in $\mathrm{ABASE}$ III named Periodicals China Base on Disc was completed by an information institute. We decided to implant records we needed from PCBD to DR database.Firstly, searched out the records relevant to rehabilitation in PCBD. Secondly, changed all these selected records from dBASE III format to ISO 2709 format. Thirdly, changed ISO 2709 format to CDS/ISIS format and implanted to DR database.

\section{Result:}

35,000 records are collected in this database. Users can search for articles, laws, papers relevant to rehabilitation published in China from 1949-1993. Every search consists of two major parts : FNDing the records and SHOWing them on the monitor.After users show the records on the monitor, users can print records and/or transfer records to floppy or hard disk.

Users can type in a search at the FND prompt, or users can select term(s) from the NDEX or word(s) and/or phrase(s) from a record displayed through the SHOW function and automatically have the system search. Type a single word in any combination of upper and lower case letter. Further narrow the search by using specially indexed limit field. Using a "limit field", the system searches only in the specified field. For example, database might have a field for publication year which has been designated as a limit field. This enables users to perform a search for a specific publication date. For the fastest search, search on the words exactly the way it appears in the INDEX.

Index collects CT, PT, authors's names, titles, journals and showed occurrences in the database . CT, PT can be corrected through their occurrences in the future. PTs with high occurrences might be $\mathrm{CT}$ in the future work. It might help indexing the documents of rehabilitation. Through database analysis, the results we can get include: (1) The process of rehabilitation development from 1949-1993 by the difference among the numbers of documents in each year. (2) Review of conditions of rehabilitation in every clinical fields. Core journals of rehabilitation are identification through database analysis. 


\section{4 . Discussion}

Two points, however, should be noted . First, the articais which we collected in DR database is an indication of relevant articles, not necessarily the degree of relevance or the quality of those articles. Second, Documents in fields of rehabilitation scattered in medical, clinical, engineering and social fields. Although we had done our best to collect these documents, it is far from complete with respect to the literature of rehabilitation.

Individuals, hospitals and institutes seeking informations relevant to rehabilitation, therefore, may find the database of use.

\section{References}

1. Richard W. Bohannon (1991), Core journal, of Rehabilitation : indentification through index analysis. International Journal of Rehabilitation Research 14,pp.333335

2. Keith Andreus (1993). Writing for medical journals. Clinical Rehabilitation 1993:7,pp.91-98 